\title{
Lexical Ambiguity
}

\author{
Jennifer Rodd
}

Department of Experimental Psychology, University College

London

j.rodd@ucl.ac.uk; @jennirodd

Chapter to be included in Oxford Handbook of Psycholinguistics. Eds M.G. Gaskell \& S-A Rueschemeyer.

March 2017 


\section{Introduction}

Most words are ambiguous: a single word form can refer to more than one different concept. For example, the word form "bark" can refer either to the noise made by a dog, or to the outer covering of a tree. This form of ambiguity is often referred to as 'lexical ambiguity'. Some researchers prefer the term 'semantic ambiguity' as this makes it clear that it is the meaning of the word that is ambiguous and not its form or grammatical properties (Vitello \& Rodd, 2015), but these terms are largely interchangeable. This chapter uses the term 'lexical ambiguity' due to its more widespread use.

Lexical ambiguity is ubiquitous. In English over $80 \%$ of common words have more than one dictionary entry (Rodd, Gaskell, \& Marslen-Wilson, 2002), with some words having very many different definitions. Take for example, the first sentence in a recent reading comprehension test given to 10/11 year old children in England (Key Stage 2 English Reading Booklet; 2016): "Dawn was casting spun-gold threads across a rosy sky over Sawubona game reserve". If we look up each of these words in a typical dictionary (Parks, Ray, \& Bland, 1998) they have, on average, 8.8 definitions per word. To correctly understand the meaning intended by the author, the reader must select a single, contextually appropriate meaning for each word. For example, they must work out that "Dawn" does not refer to a girl's name and that "game" does not refer to a form of competitive sport. And yet, despite the proliferation of ambiguity throughout this sentence, readers are usually unaware of the alternative possible meanings for such words.

One situation in which people do become explicitly aware of lexical ambiguity is when understanding puns that are deliberately constructed to make reference to both meanings of a lexically ambiguous word. For example in the joke "What did the fish say when he swam into a wall? Dam.", both meanings of the ambiguous wordform 
"dam/damn" (i.e. wall of a reservoir vs. expression of anger) are partially consistent with the sentence context and in order to understand the humour of the pun, both meanings must be accessed. But puns are the exception that prove the rule - in most circumstances listeners/readers are only aware of the contextually appropriate meaning that was intended by the speaker/author.

In summary, the language comprehension system is highly skilled at dealing with the high level of lexical ambiguity in natural language. Readers and listeners are (usually) able to find a single meaningful interpretation for each sentence that they encounter without being overtly distracted by the myriad of other possible meanings for the constituent words. This chapter will introduce the different forms that lexical ambiguity can take and explore what is known about how these words are learned, represented and processed, such that they can be rapidly and accurately understood.

\section{Different Forms of Lexical Ambiguity}

Words can be ambiguous in different ways. Sometimes, as for the word "bark", the different alternative meanings are not semantically related to each other and do not share a common origin within the history of the language. For these words, it is a historical accident that the language has evolved over time such that a single wordform corresponds to two separate, unrelated meanings. Unrelated meanings like these are usually given separate entries in dictionaries and these words are usually referred to by linguists as homonyms. However, a word like "bark" can also be referred to as a homograph, which indicates more specifically that the two meanings share their spelling. English also contains homographs where the two different meanings share only their spelling and not their pronunciation (e.g., "sow", "lead", and "close"; Figure 1). In contrast, the term homophone refers specifically to words that share their pronunciation and so includes both words like "bark" as well as words that have 
different spellings (e.g., "meet/meat", "buy/by", "there/their/they're"), and which are therefore ambiguous in spoken, but not written, language. True homonyms like "bark" are relatively rare: a dictionary count of these words revealed that only about $7 \%$ of common English words should be classified as homonyms (Rodd et al., 2002).

\title{
Homographs: \\ Words that share their spelling
}

\author{
Homophones: \\ Words that share \\ their pronunciation
}

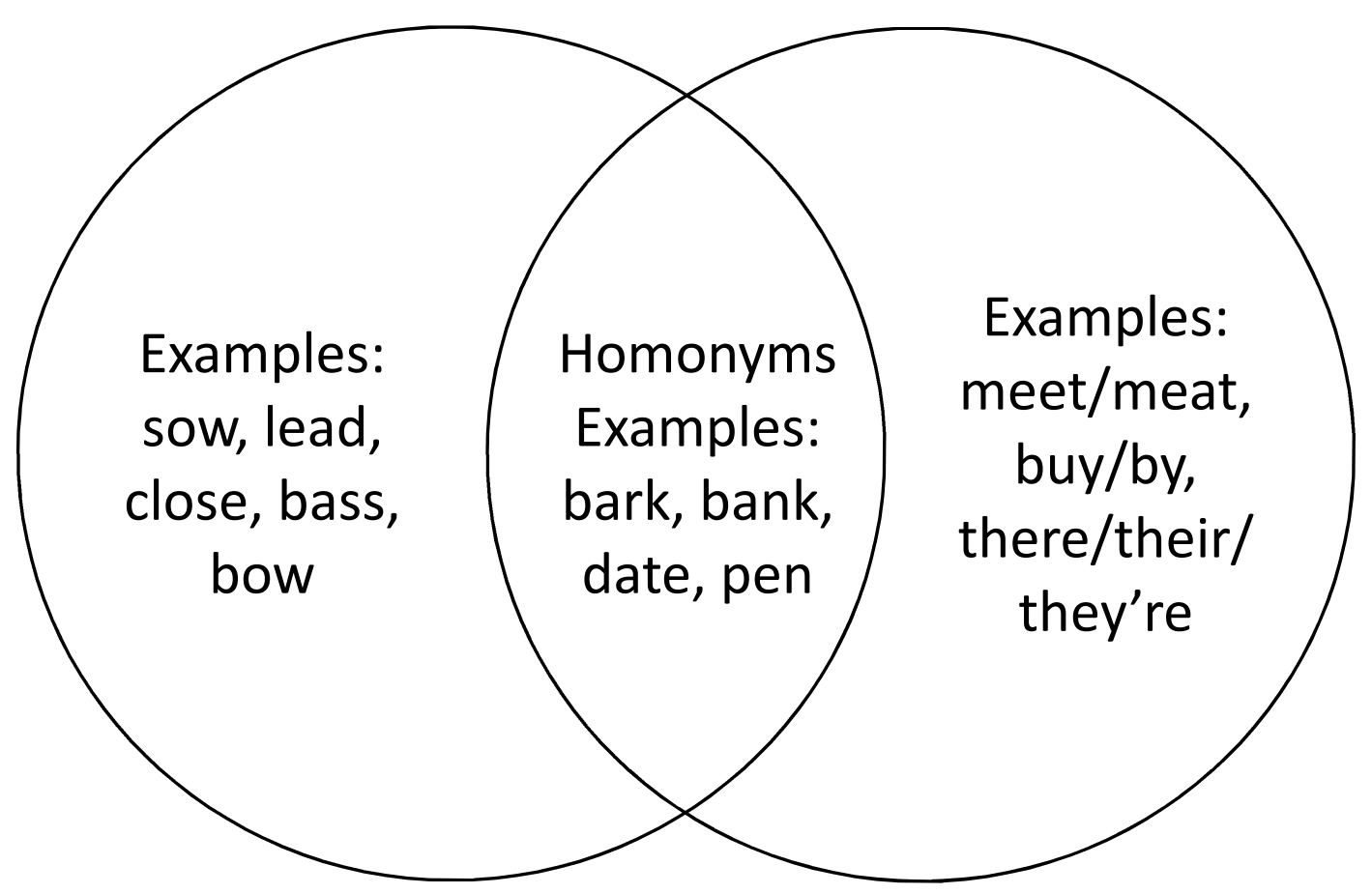

Figure 1. Terminology used to describe words with multiple different meanings

A second, and more common, form of lexical ambiguity is the ambiguity between semantically related word senses, usually referred to as polysemy. For example, the word "run" is a polysemous word with a large number of related 
dictionary definitions (e.g., "the athlete runs down the track", "the mayor runs for election", "the film runs at the cinema"). These different ways in which the word "run" can be used overlap somewhat in their meanings, but to fully understand any sentence that contains the word "run" the reader/listener must figure out exactly which definition was intended by the writer/speaker. Rodd et al. (2002) estimated that at least $80 \%$ of common words have multiple related dictionary senses. In addition, the list of word senses that we might find for a particular word in any given dictionary probably only captures a small subset of the range of ways in which that word is used in natural conversation. In contrast to homonyms, which can be viewed as a troublesome form of ambiguity that makes comprehension more difficult without any clear benefit, polysemy is of huge benefit in terms of the communicative richness of language. That words can be used in a highly flexible way to capture numerous subtly different shades of meaning is a key property of language - if we were restricted to one tightly specified meaning per word then the range of possible meanings that we could convey would be greatly reduced (see Piantadosi, Tily, \& Gibson, 2012 for further discussion).

This distinction between the ambiguity that exists between unrelated word meanings (homonymy) and related word senses (polysemy) is well established within the linguistic literature (e.g., Cruse, 1986), and is respected by the lexicographers who have created all standard dictionaries. As will be discussed in the following sections, this distinction has also been shown to have important consequences for how words are represented and processed by readers/listeners (Rodd et al., 2002; Klein \& Murphy, 2001).

Thus far, we have only considered the forms of ambiguity that exist for a monolingual speaker of English or other languages. But it is important to consider the extent to which for bilingual speakers, who know words from more than one language, 
the level of ambiguity is even higher. The impact of such cross-language ambiguity is limited by a number of factors that make individual languages different to each other. First, individual languages differ in the inventories of sounds from which their words are built. In addition, languages tend to differ in terms of the ways in which these individual sounds are combined together. For example Japanese has strict rules on how consonants can be combined, such that many English words with complex consonant clusters could not exist in Japanese. Taken together, these factors mean that many words that occur in an individual's first language could not also occur in their second language. (see Marian, Bartolotti, Chabal, \& Shook, 2012; Vitevitch, 2012 for discussion of the extent to which various European languages contain words with similar phonology/orthography)

However despite these clear differences between languages, cross-language ambiguity can occur, especially for the written forms of languages that are descended from a common ancestral language. Take for example the Dutch word "room", which translates to "cream" but shares its form with an English word with a very different meaning. These words, which are relatively rare, are known as 'false friends' or 'interlingual homographs'. Interestingly, despite the very strong contextual cues that are present during reading as to which language, and therefore which meaning, was intended this form of ambiguity can cause additional disruption for bilingual speakers (e.g., Dijkstra, Grainger, \& Van Heuven, 1999; Poort, Warren, \& Rodd, 2016). In addition Dutch-English bilinguals will also encounter cognates: words like "film" that have similar meanings in their two languages. These cognates can be relatively common in closely related languages. For example, using an automated approach that compared the spellings of word pairs that were classified as 'translation equivalents' from a database used by professional translators, Schepens, Dijkstra \& Grootjen (2012) found relatively high numbers of cognates for closely related 
languages (e.g., Dutch and German: 3785 cognates) and lower numbers for more distantly related pairs (e.g., (Spanish and German: 869 cognates). (Note that many more cognates were identified that had similar, but not identical spellings, e.g., ("idea" - "idee"). The presence of such cognates is usually viewed as helpful for readers as the two lexical items will share a common meaning and therefore if both are (partially) activated in parallel this would help rather than hinder the readers.

Finally, it is important to note that lexical ambiguity is not a static phenomenon. Any individual speaker of a language will continue to gain new meanings/senses for the words that they already know throughout their lifetime. Both children and adults continue to learn meanings/senses that they did not previously know, for example because they have taken up a new hobby, career or academic subject (e.g., the sailing related meaning of "boom" or the statistical meaning of "normal"). In addition, individuals will also need to learn new meanings/senses that are added to the language over time due, for example, to technological developments such as the social media senses of "tweet", "post" and "friend" (Rodd et al., 2002; Blank, 1999).

In summary, lexical ambiguity is a ubiquitous phenomenon: most of the words that we use can refer to more than one possible concept. Although levels of lexical ambiguity can differ across languages (Bates, Devescovi, \& Wulfeck, 2001), lexical ambiguity is the norm and not the exception. Therefore any general account of how word meanings are represented and accessed must incorporate an explanation of meaning representation and access for ambiguous words.

\section{How are ambiguous words represented?}

Over the past 50 years, psycholinguists have debated how ambiguous words are represented within the 'mental lexicon', the memory store that contains information about all the words that an individual person knows. An early experimental 
finding that was highly influential in framing this discussion was the 'ambiguity advantage' in the visual lexical decision task. This task, which is a staple of psycholinguistic research, requires participants to decide, as quickly and accurately as possible, whether each string of letters that they see is a real word in their language (e.g., "hat") or a made-up non-word (e.g., "wug"). A relatively large set of studies reported faster visual lexical decisions for ambiguous words compared with unambiguous words (Rubenstein, Garfield, \& Millikan, 1970; Jastrzembski, 1981; Azuma \& Van Orden, 1997; Borowsky \& Masson, 1996; Hino \& Lupker, 1996; Kellas, Ferraro, \& Simpson, 1988; Millis \& Button, 1989; Pexman \& Lupker, 1999).

Early explanations for this 'ambiguity advantage' were framed within a view of the mental lexicon that can broadly be characterised as the 'localist' approach. Models of this type assume that each word that we know is represented by a single entry in our mental lexicon. Within the connectionist framework these entries correspond to individual 'nodes' or 'units'. (See the Interactive Activation and Competition Model (McClelland \& Rumelhart, 1981) and TRACE (McClelland \& Elman, 1986) for influential models of written and spoken word recognition that include such localist representations.) Within this framework, these localist word units are seen as the gateway to information about a words meaning: as soon as one of these units has been sufficiently activated in response to the incoming visual/auditory input the reader/listener can then retrieve all the information about that word's meaning (and its grammatical properties). Researchers using lexical decision tasks commonly assumed that participants were able to make a 'yes' response as soon as one of these localist word units reached some threshold level of activation. Within this framework a common interpretation of this ambiguity advantage, was that ambiguous words benefit from having multiple entries within the mental lexicon (Figure 2a). For example, Jastrzembski (1981) suggested that ambiguity advantage arises because 
of the noise that is present in the system and that can produce random fluctuations in the degree to which individual units are activated during word recognition: because ambiguous words have more than one 'competitor' in the race for recognition, on average by any given point in time, one of the two competitors is more likely to have reached the threshold for recognition compared with an unambiguous word with only one competitor. Thus the ambiguity advantage was taken as evidence for the claim that words are represented as localist word units, and that ambiguous words have multiple separate representations - one for each meaning. 
(a) Localist Representations of Ambiguous words

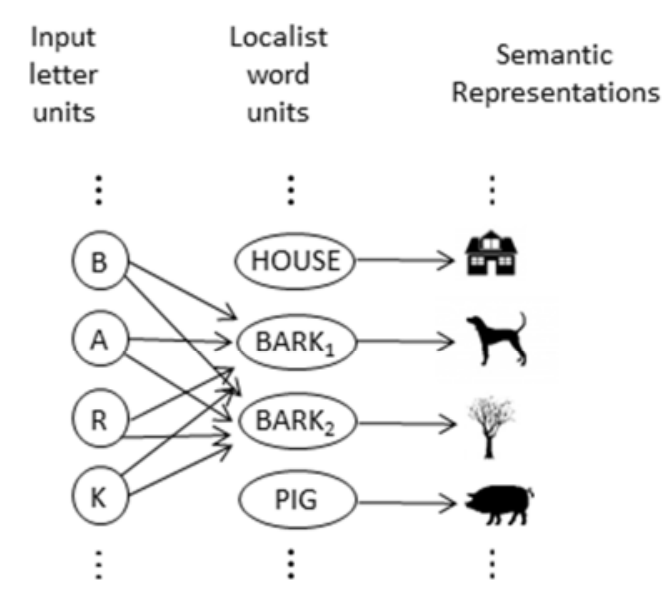

(b) Distributed Representations of Ambiguous words

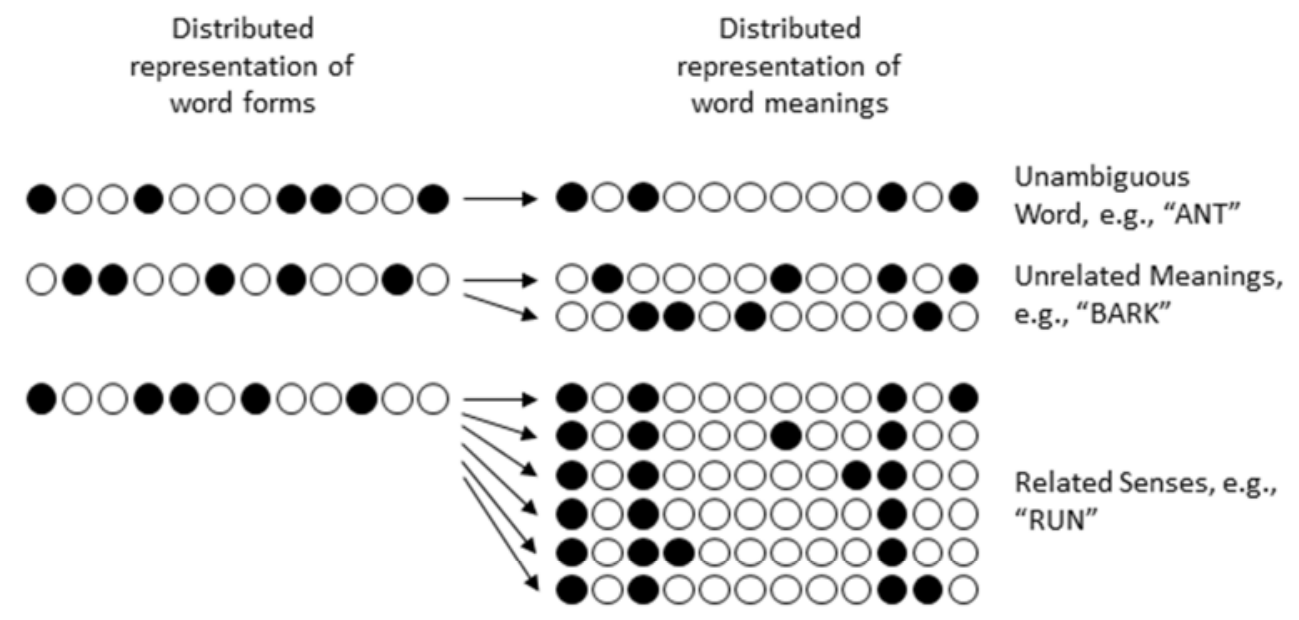

Figure 2. Localist and distributed views on how ambiguous words are represented in the mental lexicon 
However subsequent research suggested that this early view was too simplistic. First, it became clear that the 'one unit per meaning' approach could not fully explain the different types of ambiguity that exist. While this approach is plausible for words with completely distinct, unrelated meanings (e.g., "bark"), it was far from clear how words with large clusters of highly related word senses (e.g., "run") should be represented within such a scheme, or how the system would distinguish between these two forms of ambiguity - many words have meanings that are intermediate in relatedness between these two extremes. In contrast, some authors argued that this variability in the relatedness of word meanings can be captured more naturally by an alternative, and conceptually very different, view of word recognition, often referred to as the 'distributed' approach (Rodd, Gaskell, \& Marslen-Wilson, 2004). This approach abandons the idea that words are represented by single 'localist' entries in the mental lexicon and instead assumes that each word that we know is represented as a unique pattern of activation across sets of units that collectively represent its form (i.e. spelling/orthography or sound/phonology) and its meaning (Figure $2 b$ ). These units can be thought of as representing different features of the words' form/meaning. Words with similar spelling (or sound) will activate similar sets of orthographic (or phonological) input units, while words with similar meanings will activate similar sets of semantic units (see Gaskell \& Marslen-Wilson, 1997; Hinton \& Shallice, 1991; Joordens \& Besner, 1994; Plaut, 1997; Plaut \& Shallice, 1993 for models of this type). Within this distributed framework, the single orthographic (or phonological) input pattern for an ambiguous word must map onto multiple different semantic patterns that correspond to its different meanings or senses. For example, a word like "bark" would map onto two completely uncorrelated patterns of semantic activation, whereas a word like "run" will correspond to a large number of highly correlated semantic patterns (Figure 2b). 
Although this 'distributed' view of the mental lexicon seemed highly plausible in terms of its capacity to represent the wide variety of ambiguous words that exist in natural language, the finding of a benefit in lexical decision for ambiguous words seemed hard to explain within this framework. When the orthographic (or phonological) pattern is presented to the network, the model will attempt to simultaneously instantiate both of the word's meanings across the same set of semantic units. These competing semantic representations are likely therefore interfere with each other, and this interference effect means that models of this type generally predict slower recognition of words that are lexically ambiguous. (Although see Joordens \& Besner, 1994; Borowsky \& Masson, 1996; Kawamoto, Farrar, \& Kello, 1994 for attempts to explain how an ambiguity advantage might arise; see Rodd et al., 2004 for detailed discussion.)

This apparent discrepancy between the prediction that the meanings of ambiguous words should interfere with each other during recognition, and the reported 'ambiguity advantage' in visual lexical decision tasks was addressed by Rodd, Gaskell, and Marslen-Wilson (2002), who argued that the previous studies did not adequately take account of the distinction discussed above between homonyms like "bark" that have two semantically unrelated meanings, and polysemous words like "run" that have a collection of semantically related word senses. They argued that the lexical-semantic representations of these different types of words will be very different and that the two types of ambiguity could potentially have different effects on how easily a word's meaning is accessed. In a set of visual and auditory lexical decision experiments, Rodd, Gaskell, and Marslen-Wilson (2002) only replicated the classic ambiguity advantage for words that have many highly related word senses (e.g., "run"). In contrast, for words with multiple unrelated meanings (e.g., bark), they 
found the reverse effect: an ambiguity disadvantage. They suggested that the prevalence of an advantage for ambiguity in the earlier experiments reflected the fact that ambiguity between related word senses is very much more frequent than ambiguity between unrelated word meanings, and that previous studies tended to select more of the former word types within their category of lexically ambiguous words (see Beretta, Fiorentino, \& Poeppel, 2005; Klepousniotou \& Baum, 2007 for replications of this finding; see Rodd et al., 2012 for an analogous effect for newly learned word senses/meanings; see Rodd, 2004 for a sense benefit in word naming for words with inconsistent spelling).

The observed disadvantage for words with multiple unrelated meanings can easily be accommodated by distributed connectionist models. As described above, these models assume that the unrelated, uncorrelated meanings of these words will interfere with each other during meaning access and this interference delays the point in time at which the participant has retrieved a sufficiently stable representation to be able to confidently press the 'yes' button during the lexical decision task. Rodd, Gaskell, Marslen-Wilson (2004) implemented a connectionist model of this type and confirmed that this form of competition to access a single coherent word meaning does indeed produce slower retrieval for the meanings of ambiguous words. This model was also able to provide an account of the benefit for words with multiple senses (i.e. polysemous words). In their implementation of the model, the authors assumed that these words, which have partially overlapping meanings, are represented by a range of different, but highly correlated, patterns of activation across the semantic units such that only a subset of the word's possible semantic features are present for any specific word sense. This variability in the precise semantic representation of these polysemous words resulted in the formation of highly robust representations as the network learned these words. Importantly, they argued that 
this benefit for words with multiple senses should only be seen on tasks such as lexical decision in which it is not necessary to retrieve a very specific sense, but rather where it is sufficient to retrieve a very general 'blend' of its different meanings.

However, it is important to note that while this model adequately explains the patterns of behaviour seen in lexical decision tasks by assuming that these reflect important differences in the speed of access to different types of word meanings, there has subsequently been some disagreement about whether these differences arise because of competition at the level of decision making (Hino, Pexman, \& Lupker, 2006) or at the level of lexical-semantic processing (Armstrong \& Plaut, 2016). Future work is still needed to clarify the extent to which these effects are being driven by lexical-semantic or decision-level effects.

\section{How are ambiguous meanings learned?}

The studies described above focus on how words that have multiple meanings are represented within the mental lexicon of adults for whom both meanings are already highly familiar. This naturally leads to the question of how these meanings are learned in the first place, by both children and adults. Most studies on this topic have focused on the situation where one meaning is already highly familiar, and within the course of the experiment the participants are required to learn an additional (often fictitious) meaning for these familiar words. This is akin to the situation that is frequently faced by adult learners when they encounter a new meaning that has recently entered the language, or the specialist vocabulary of a new academic subject or hobby (e.g, a rower learning that "feather" refers to a position of their oar; Rodd et al., 2016).

Several studies using this approach have shown that children (age 3-10) find it relatively difficult to assign new meanings for words that they already know (Casenhiser, 2005; Doherty, 2004; Mazzocco, 1997). For example, they find it more 
difficult to learn that the familiar word such as "spade" refers to an unfamiliar novel object compared with assigning an entirely unfamiliar wordform to this novel meaning. These studies are consistent with the view that children find it easiest to learn the mapping from form to meaning when this mapping is 'one-to-one' (i.e. each form maps on to only one meaning), and that 'one-to-many' mappings are intrinsically more difficult to learn due to interference or competition from the alternative meaning (see Dautriche \& Chemla, 2016 for further discussion of why this difficulty arises; see Rodd et al., 2004 for details of how this effect is explained within a connectionist modelling framework). In contrast, data from Storkel and colleagues (Storkel, Maekawa, \& Aschenbrenner, 2013; Storkel \& Maekawa, 2005) suggests that children find it easier to learn new meanings for familiar words (compared to completely novel formmeaning pairs) because in the latter situation the child has the additional demand of having to learn a new word form.

Recent studies with adults have pointed towards one explanation for these apparently contradictory findings; Fang, Perfetti \& Stafura (2016) suggest that two different factors play an important role in determining whether it is easier to learn a new meaning for a familiar or unfamiliar word. First, they suggest that for familiar words, learners benefit from a relatively short-lived boost in learning that is due to their existing familiarity with the wordform, which is already in their mental lexicon and does not need to be newly learned. This benefit ceases to be present once learners have become sufficiently familiar with the new word forms. But, crucially, learning of new meanings for familiar words is also made more challenging due to interference from the alternative, familiar meaning. Fang, Perfetti \& Stafura suggest that although this lexical-semantic interference is present throughout learning, it only becomes evident once the benefit from familiarity has diminished. Thus the pattern of results 
that are observed in any given experiment will depend on how well the meanings are learned when the individual is tested.

Studies of word-meaning learning with adult learners have also revealed that not all ambiguous words are equally difficult to learn. In particular, adults find it easier to learn new meanings for existing words, when the new meaning is semantically related to the existing meaning (e.g., that "ant" refers to a very small, mobile listening device) compared with a completely unrelated meaning (Rodd et al., 2012). This finding suggests that partial overlap between the old and new meanings can facilitate learning. Given the finding that ambiguity between multiple related word senses is far more common than ambiguity between unrelated word meanings (Rodd et al., 2002), this finding helps to explain how word meanings such as the social media meanings of "tweet" and "troll" appear to enter the language relatively easily: acquisition of such word meanings is facilitated by their semantic relationship with the existing familiar meaning.

In addition to the studies described above that require participants to learn just one novel meaning for a familiar word, a smaller set of studies have explored the situation in which the learner is simultaneously exposed to two different meanings for a novel word form. This situation is more akin to the challenge facing a young child in the early stages of language acquisition where they hear, for example, the word "bark" being used to refer to two very different concepts in the world, and where they are still relatively uncertain about both meanings. These studies show that despite the additional challenges associated with learning ambiguous words, it is clear that children (and adults) can (and frequently do) learn that words can have multiple meanings. For example. when children are exposed to two new meanings for a novel wordform that are semantically distinct (e.g., the wordform "blicket" is used to refer to both snakes and monkeys), they tend to assume that the wordform is ambiguous 
between these two relatively specific meanings, and do not usually assume that it corresponds to some very general superordinate meaning that also encompasses all other animals (Dautriche, Chemla, \& Christophe, 2016; Dautriche \& Chemla, 2016).

There is also evidence that children as young as four can benefit from a relatively sophisticated understanding of how some forms of ambiguity can be highly systematic. For example, Srinivasan \& Snedeker (2011) showed that when four-yearold children were taught that a novel wordform (e.g., "blicket") refers to the physical object of a book, they readily understood this wordform can also be used to refer to the contents of the book (e.g., "the shiny blicket" vs "the interesting blicket"). In contrast, they showed no such extension between the two meanings of a homograph, such as "bat": if they were taught that "blicket" referred to the flying mammal meaning of "bat" they did not readily assume that it also meant a "baseball bat". Studies have also shown that adult participants are highly skilled at working out the meanings of novel word senses (for example, using the name of a famous person to refer to a book they have written; Clark \& Gerrig, 1983; Frisson \& Pickering, 2007). There is also evidence that children systematically overestimate the range of possible senses that a familiar word can have, for example considering that the word "movie" can refer to the disc on which it is stored, a meaning that adults would usually consider unlicensed (Rabagliati, Marcus, \& Pylkkänen, 2010).

Taken together, these results show that although lexical ambiguity can make word learning significantly more difficult, both children and adults are able to learn the meanings of ambiguous words by being highly sensitive to the precise contexts in which words are used. However, it remains a challenge for models of word learning to explain the precise mechanism(s) by which ambiguous words are learned. Many influential models of word meaning learning focus primarily on how unambiguous 
words are learned and do not make explicit claims about how word learners deal with lexical ambiguity (see Dautriche et al., 2016 for extensive discussion).

\section{Understanding ambiguous words in sentences}

While tasks using single words presented in isolation (e.g., Rodd et al., 2002) provide important insights into how lexically ambiguous words might be represented, to understand how these words are processed in natural language requires experiments in which they are presented within sentence contexts. Understanding the mechanisms by which only the contextually appropriate meaning is selected for each word from the range of possible word meanings is a necessary component of any model of language comprehension. Such a model must explain how, for example, the word "bark" can be interpreted differently in sentences like "the girl saw the bark" compared with "the girl heard the bark". Without such a disambiguation mechanism, virtually any sentence in natural language would be impossible to understand accurately.

Although there is some disagreement on the details of how disambiguation proceeds, the literature has converged on the view that whenever a listener (or reader) encounters an ambiguous word (e.g., "bark") they rapidly and automatically retrieve in parallel, at least to some extent, all the meanings that they know for this word (e.g., "dog noise", "tree covering"), and then, within a few hundred milliseconds, select the single meaning that is most likely to be correct (Duffy, Morris, \& Rayner, 1988; Rodd, Johnsrude, \& Davis, 2010; Simpson \& Kang, 1994; Twilley \& Dixon, 2000). This view is somewhat at odds with most people's introspection - most people feel that they are able to directly access the correct meaning of each word that they encounter, and don't have any conscious experience of retrieving and then having to reject inappropriate meanings. But the experimental literature indicates that there is 
indeed transient, albeit largely unconscious, access of multiple meanings for ambiguous words during sentence comprehension.

This view that we retrieve multiple meanings and then select just one of them, is often referred to as the "exhaustive access" model, because the listener/reader 'exhaustively' retrieves all possible meanings. Initial support for this claim came from experiments using the cross-modal semantic priming paradigm. In this paradigm, participants first hear an ambiguous word (e.g., "bug") in a sentence context, and then make lexical decision responses to visual probe words that are related to one of other of its meanings (e.g., "spy" vs. "ant"). If people are faster at making lexical decisions to these probe words compared with an unrelated word (e.g., "sew") then this is taken as evidence that they have accessed the relevant meanings of the initial prime word (i.e. "bug"). Researchers using this paradigm found that even when the ambiguous word (e.g., "bug") is heard within a sentence that was only consistent with one of the meanings (e.g., "The man was not surprised when he found several spiders, roaches, and other bugs"), if the visual probe appears immediately after the ambiguous word, then responses were faster for probes that were semantically related to either the contextually appropriate meaning (e.g., "ANT") or to the inappropriate word meaning (e.g., "SPY") (Onifer \& Swinney, 1981; Swinney, Onifer, Prather, \& Hirshkowitz, 1979). This finding indicates that both these meanings had been automatically activated despite the fact that the preceding words provided enough information to rule out one of these meanings. In contrast, if the visual probe appears a short while after the ambiguous word (e.g., 3 syllables in Swinney, 1979), then faster responses are only seen for the contextually appropriate meaning (i.e. "ANT" but not "SPY"). This latter finding indicates that listeners are able to select the more appropriate meaning relatively quickly, such that any other meanings are no longer active. 
Following on from these early cross-modal priming experiments, other researchers have shown that, for sentences in which the words that come before the ambiguous words are very strongly constrained towards the dominant (more frequent) meaning (e.g., "the violent hurricane did not damage the ships which were in the PORT"), then only the contextually appropriate meaning appears to be active (Tabossi, 1988; Tabossi \& Zardon, 1993). Together with convergent evidence from experiments that monitor how readers' eye-movement change when they encounter an ambiguous word, this evidence supports the view that when readers/listeners encounter an ambiguous word, its multiple meanings are usually activated in parallel but that the level of this activation is influenced by two key factors, sentence context and meaning frequency, such that meanings that are highly frequent or very strongly supported by the preceding context are more readily available (MacDonald, Pearlmutter, \& Seidenberg, 1994; Simpson \& Kang, 1994; Twilley, Dixon, Taylor, \& Clark, 1994). This view is exemplified in the highly influential "reordered access" model of ambiguity resolution (Duffy et al., 1988).

So far we have just considered the case where the preceding sentence context provides a strong cue as to which word meaning is correct, but cross-modal priming experiments have also provided insights into what occurs when the preceding context is consistent with multiple meanings (e.g., "the woman noticed the bark"). There are, logically, two possible strategies when faced with such a sentence. Either the listener/reader maintains the multiple possible interpretations in parallel until the point where there is enough information to rule out one of the options, or they make a 'best guess' about which meaning is more likely to be correct, and then if necessary reinterpret the sentence if this guess turns out to be incorrect. The results from early cross-modal priming studies are more consistent with the latter view. These studies found that listeners do not maintain multiple meanings for long but instead make a 
rapid selection within a few hundred milliseconds of encountering an ambiguous word (e.g., Seidenberg, Tanenhaus, Leiman, \& Bienkowski, 1982; Swinney et al., 1979). Seidenberg et al. (1982) proposed that this strategy is used because limits on processing capacity make it difficult to maintain multiple interpretations of sentences in parallel (but see Mason \& Just, 2007; Miyake, Just, \& Carpenter, 1994). In the absence of strong sentence context that could indicate which meaning is most likely to be correct, research indicates that the primary cue used by readers/listeners to make their 'best guess' is meaning dominance, that is, the relative frequencies of the different meanings (Twilley et al., 1994; Armstrong, Tokowicz, \& Plaut, 2012). All other things being equal the most frequent meaning will be selected (e.g., the "writing implement" meaning of "pen", not the "animal enclosure" meaning).

More recent studies have additionally highlighted the contribution of recent experience, demonstrating that we are biased to select recently-encountered meanings. For example when we encounter an ambiguous word like "pen" without any sentence context, we are more likely to retrieve the lower frequency (subordinate) meaning if we had already encountered this meaning twenty minutes earlier (Rodd, Lopez Cutrin, Kirsch, Millar, \& Davis, 2013). This finding, known as word-meaning priming, has been shown both in laboratory studies and in more naturalistic settings where individuals encounter the target ambiguous words in subordinate meaning contexts either as part of a radio programme or while participating in their usual hobby (Rodd et al., 2013; Rodd et al., 2016). This finding that access to word meanings can by modulated by recent experience has even been shown across languages - earlier experience with the Dutch meaning of "room" can make it harder to then access the unrelated English meaning of this word form (compared to a word like "film" that has a similar meaning in both languages; Poort et al., 2016). 
Of course, the consequence of this 'early selection' strategy, where a 'best guess' is made on the basis of meaning frequency or recent experience, is that inevitably sometimes the wrong meaning will be selected. This situation is perhaps most common when the lexically ambiguous word is preceded by a neutral context and is later followed by context that does not support the expected, 'best guess' meaning. For example, if a listener was to hear the sentence "he mentioned that he'd been to the bank of the river" they would be likely to initially select the more common but incorrect, financial institution, meaning of "bank". This initial, incorrect, selection will then cause problems when the listener gets to the word "river", as this word is incompatible with the meaning of "bank" that they have selected. Thus the listener will have to revisit their initial interpretation of the sentence in order to find a word meaning that is compatible with this later information. Experiments using a wide range of methods have shown that this reinterpretation process can be very cognitively demanding. In the case of printed sentences, studies have shown that reading times are particularly long for such sentences, with readers being very likely to refixate on earlier parts of the sentences as they try to find an appropriate interpretation (Duffy et al., 1988; Kambe, Rayner, \& Duffy, 2001). For spoken sentences, the additional processing load has been revealed by dual-task paradigms in which participants listen to sentences while performing an unrelated concurrent task (e.g., detecting whether a word on a screen is in upper or lower case). Performance on this unrelated task has been shown to decline when the sentence is likely to require reinterpretation, compared with a relatively unambiguous sentence, presumably because of the additional resources being used to reinterpret the sentence (Rodd et al., 2010). In addition, numerous functional magnetic resonance imaging (fMRI) studies have shown an increase in blood flow to left frontal and temporal brain regions that occurs when a sentence requiring reinterpretation is encountered either in the written (Mason 
\& Just, 2007; Zempleni, Renken, Hoeks, Hoogduin, \& Stowe, 2007) or spoken modality (Rodd, Johnsrude, \& Davis, 2012; Vitello, Warren, Devlin, \& Rodd, 2014).

In summary, research using both spoken and written sentences and using a range of different experimental methods has converged on the view that whenever an ambiguous word is encountered, multiple meanings are initially activated and a 'best guess' is then rapidly made about which meaning is most likely to be correct. This 'best guess' is driven primarily by the immediate sentence context, but is also influenced by the overall frequency (dominance) of the word's different meanings, and by the listener/reader's recent experience with the ambiguous word. In contrast to this general agreement about how ambiguity resolution proceeds, there is, as yet, no agreement about the exact fate of any non-selected meanings. It is unclear whether the non-selected meanings are completely suppressed in order to prevent them interfering with subsequent processing (MacDonald et al., 1994), or whether they retain a low level of activation such that they can be more easily reactivated if the initial interpretation proves to be incorrect (McRae, Spivey-Knowlton, \& Tanenhaus, 1998).

As well as the wealth of studies exploring how skilled adult comprehenders process words with multiple meanings, there is a smaller, but important, set of studies looking at how the ability to process ambiguous words develops during childhood, and on how this ability relates to individual differences in comprehension skill. Broadly speaking, the literature has converged on the view that young children use a similar approach to adults when processing ambiguous words: they make relatively rapid guesses about how best to interpret each ambiguous word based on all the evidence that is currently available to them, and do not hold off on interpreting ambiguities until the end of the sentence (see Rabagliati, Pylkkänen, \& Marcus, 2013 for review). For example, Rabagliati et al., (2013) have shown that children as young as four can 
perform relatively well on a task that requires them to understand phrases like "Snoopy chased/swung the BAT" in which a single word indicates which meaning of the target ambiguous word is correct. In addition, they showed that children don't rely solely on strong lexical associations between the individual words in the sentences to decide which meaning to select, but they can also make use of a more global assessment of which meaning is most plausible in the sentence context. However, it is worth noting that these young children did make many more errors than adults in understanding these ambiguous words, suggesting that the ability to disambiguate ambiguous words rapidly and accurately can be relatively challenging for young children.

Several studies have shown that children who perform relatively poorly on general tests of comprehension skill also perform poorly on tasks that specifically involve resolving lexical ambiguities, compared to more skilled comprehenders. These studies have shown that difficulties faced by poor comprehenders when processing lexical ambiguities is likely to be caused, at least in part, by children's relatively weak lexical-semantic representations, especially for the lower frequency meanings (Henderson, Snowling, \& Clarke, 2013). In addition, difficulties in understanding low-frequency meanings of ambiguous words can also reflect weaknesses in the executive control processes that are needed to suppress/inhibit the more frequent meaning of an ambiguous word. This link between ambiguity resolution and executive function has been shown for non-clinical samples of both children (Khanna \& Boland, 2010) and adults (Gernsbacher \& Faust, 1991; Gernsbacher, Varner, \& Faust, 1990). In addition, van der Schoot et al. showed that poor comprehenders (age 10-12) found ambiguous words particularly challenging when the disambiguating information occurred after the ambiguity (van der Schoot, Vasbinder, Horsley, Reijntjes, \& van Lieshout, 2009), suggesting that less skilled 
comprehenders monitor their comprehension less effectively that more skilled comprehenders. Finally, Norbury (2005) has shown that children (age 9-17) with diagnosed language impairments are not able to use context as efficiently as control participants. Thus it seems that difficulties in understanding sentences with ambiguous words is likely to be a key factor that can limit an individual's ability to rapidly and fluently comprehend language. Further research is needed to better understand the complex causes of these difficulties and how these skills might best be improved.

\section{The Brain Mechanisms of Ambiguity Resolution}

Over the past 20 years, researchers have increasingly made use of brain imaging methods to study sentence comprehension (Rodd, Vitello, Woollams, \& Adank, 2015; Hagoort \& Indefrey, 2014). These studies have given key insights, not only into which brain regions are involved in understanding lexically ambiguous words, but also into questions about the nature of the cognitive mechanisms involved, and in particular, how these disambiguation processes relate to other aspects of comprehension.

The results from the set of published studies that have used fMRI to study how lexically ambiguous words are processed have been remarkably consistent, and have highlighted three, spatially distinct brain regions that show an increased haemodynamic response (i.e. increased blood flow) for high-ambiguity sentences compared with closely matched low-ambiguity sentences: the left inferior frontal gyrus (pars opercularis; pars triangularis) and the left posterior temporal cortex (especially the left inferior temporal gyrus and middle temporal gyrus), and (to a lesser extent) the right inferior frontal gyrus. (Figure 3; Bekinschtein, Davis, Rodd, \& Owen, 2011; Davis et al., 2007; Mason \& Just, 2007; Rodd, Davis, \& Johnsrude, 2005; Rodd et al., 2012; Rodd, Longe, Randall, \& Tyler, 2010; Zempleni et al., 2007; Tahmasebi et al., 
2012: See Vitello \& Rodd (2015) for recent review and Rodd et al (2015) for formal meta-analyses that includes these studies).

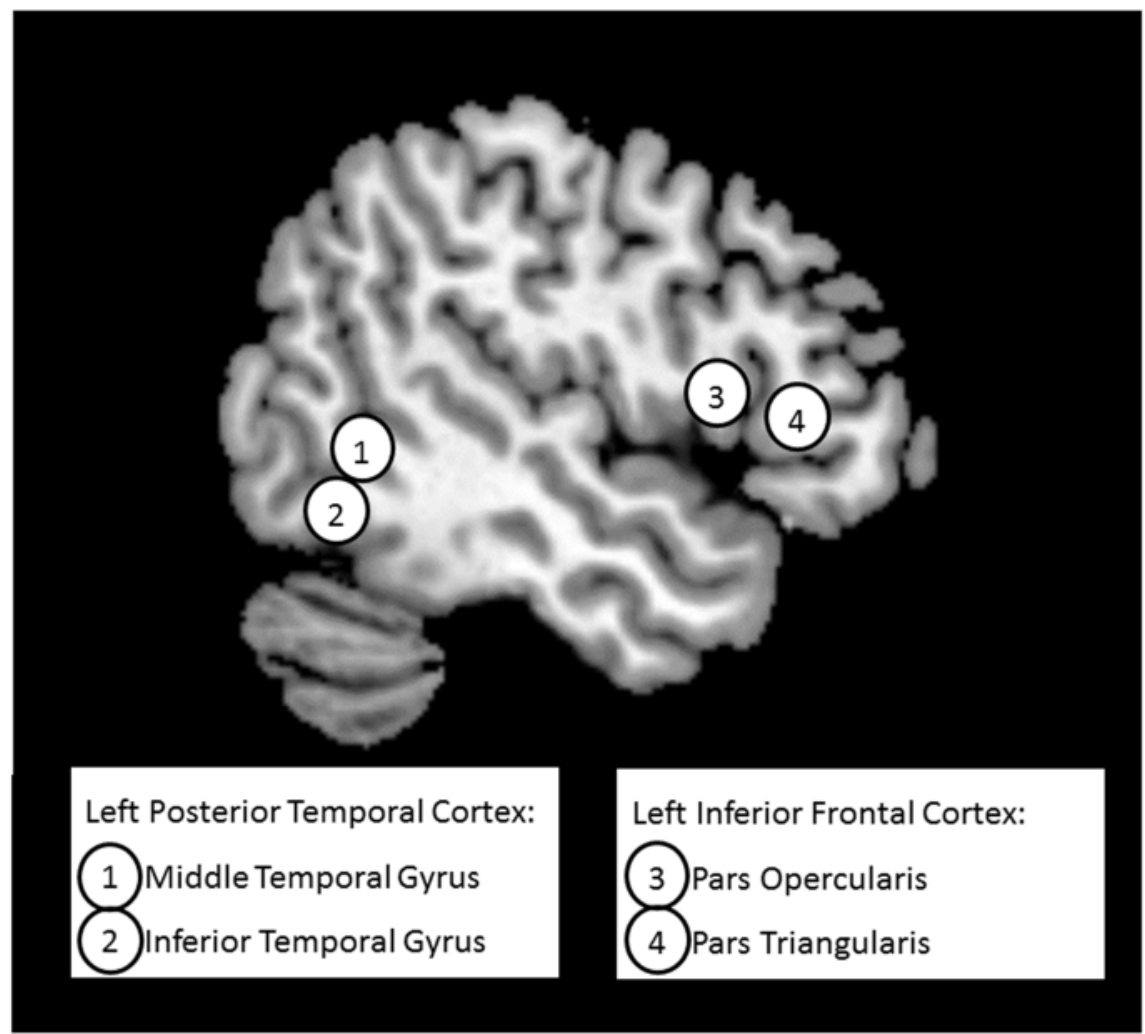

Figure 3: Left hemisphere regions that show increased activation for sentences containing lexical ambiguities (shown on structural scan from single participant (Rorden \& Brett, 2000).

Of the three brain regions associated with the processing of sentences containing lexical ambiguities, the most well-studied and best understood is the left inferior frontal gyrus (LIFG), more specifically the posterior and middle sub-divisions of the LIFG (pars triangularis; pars opercularis). This region of the posterior frontal lobe is sometimes referred to as 'Broca's Area' due its association with Paul Broca who 
reported language impairments in individuals with damage to this region. Not only has this area been very consistently activated in all studies that have used sentences containing lexical ambiguities (see Vitello \& Rodd, 2015 for a recent review), but a recent study has also confirmed that recruitment of this region is highly consistent across individuals: 95\% of participants showed an ambiguity-related response in close proximity to the group LIFG peak (Vitello et al., 2014).

Rodd et al. (2012) provided evidence that this region supports multiple aspects of disambiguation, including both the initial selection between alternative interpretations when an ambiguous word is first encountered, as well as any subsequent reinterpretation if the wrong meaning was initially chosen. Rodd et al. (2012) found activation in this region for spoken sentences in which the disambiguating information came before the ambiguous word (e.g., "the hunter thought that the hare/hair in the field was actually a rabbit"). Because these sentences used the lower frequency meaning of all the ambiguous words, it was relatively difficult for listeners to select the correct meaning for the ambiguous word when they first encountered it, but because of the strongly constraining context that that preceded the ambiguity, it is highly likely that the correct meaning would be selected and that no later reinterpretation would be required. Thus the greater LIFG activation that was seen for these sentences, compared with low-ambiguity controls, is most likely to reflect the involvement of this region in the initial selection of the ambiguous word's meaning. LIFG activation was also seen for sentences in which the critical disambiguating information occurred a few words after the ambiguous word (e.g., "the scientist thought that the film on the water was from the pollution"). An analysis of the time-course of activation for sentences that varied in the position of the disambiguating words confirmed that this activation was driven primarily by the disambiguating information and not by the ambiguity itself, providing strong evidence 
for the involvement of this region in sentence reinterpretation (See also Mason \& Just, 2007; Zempleni et al., 2007; Vitello et al., 2014 for evidence of LIFG involvement in reinterpretation.).

Importantly, although the LIFG is clearly important for processing lexical ambiguities, it does not seem to be specialised for this form of linguistic processing. First, within the field of sentence comprehension Rodd et al. (2010) found that the same posterior LIFG region (peak in pars opercularis) was activated for both lexical ambiguities and for syntactically ambiguous sentences (e.g., "visiting relatives is/are..."). This indicates that this region provides a relatively general processing resource for resolving linguistic ambiguities, regardless of the specific linguistic nature of the ambiguity. In addition, activation in this region has been reported in numerous studies using a very diverse range of sentence types and experimental manipulations that require additional sentence-level processing, such as semantic/syntactic anomalies or complex grammatical structures (see Rodd et al., 2015 for detailed review and formal meta-analysis). Perhaps most importantly, activation in the posterior LIFG has been seen in numerous studies using non-sentence stimuli, such as single words or pictures (e.g., Bedny, McGill, \& Thompson-Schill, 2008; Whitney, Kirk, O'Sullivan, Lambon Ralph, \& Jefferies, 2011; Kan \& Thompson-Schill, 2004; Thompson-Schill, D'Esposito, Aguirre, \& Farah, 1997), emphasising that its role is not confined to sentence processing.

In response to this heterogeneity in the types of studies that produce activation in the LIFG, several important theoretical accounts of its function have been proposed. A neurobiological model of language put forward by Hagoort and colleagues (Hagoort, 2005; Hagoort \& Indefrey, 2014) subdivides language processing into three components: Memory, Unification, and Control. Within this theory, the LIFG constitutes a unification space which allows basic units of linguistic 
information to be combined together to form more complex representations of individual sentences and of longer pieces of discourse. Under this view, it is the increased combinatorial demands of high-ambiguity sentences that drives greater LIFG activation for these sentences, as well as for sentences that contain anomalies or that use complex grammatical structures.

In contrast to this 'unification' account of LIFG function, which focuses primarily on sentence comprehension, an alternative neuro-cognitive account of LIFG function, known as the "conflict resolution account" (Novick, Kan, Trueswell, \& Thompson-Schill, 2009; Novick, Trueswell, \& Thompson-Schill, 2005; ThompsonSchill et al., 1997), takes a broader view and argues that the key function of the LIFG is to resolve competition between activated representations for both sentence and non-sentential stimuli. Specifically, Novick et al. (2009) suggested that the LIFG supports conflict resolution either when there is a prepotent but irrelevant response, or when multiple representations are available but no dominant response exists. Although this account can easily accommodate the findings from the lexical ambiguity literature - activation increases when the word's strongly dominant (i.e. prepotent) meaning is not consistent with the sentence context (e.g., "the sheep was in the pen") or when two equally likely meanings are both consistent with a relatively neutral context (e.g., "he mentioned the organ").

In summary, different theories of LIFG function can explain the involvement of this region in ambiguity resolution in terms of a relatively general role in either combinatorial processing or conflict resolution. These are both cognitive functions that are not specifically restricted to the processing of lexical ambiguities and both accounts emphasize the involvement of relatively generic forms of cognitive processing in resolving these ambiguities. This emphasis in the neuro-cognitive models is in contrast to the psycholinguistic models reviewed above, which focus 
more on specifying the specific processes used within processing of lexical ambiguities.

Finally, as previously noted, it important to recall that lexical ambiguities also produce consistent activation in the posterior portion of the left temporal lobe (Davis et al., 2007; Rodd et al., 2005; Rodd et al., 2012; Zempleni et al., 2007; Tahmasebi et al., 2012), and (less consistently) in the right IFG (Mason \& Just, 2007; Rodd et al., 2012; Zempleni et al., 2007). Unfortunately, in stark contrast with the literature on LIFG function, the precise locations of these activations is highly variable, and the field has made relatively little progress in specifying the precise contributions of these regions and how they relate to the literature beyond lexical ambiguity. Thus at the current time, although it seems clear that both of these regions make an important contribution to the processing of lexical ambiguities, future research is needed to specify the cognitive nature of this contribution (see Vitello et al., 2014 for a more extensive review).

\section{Conclusions}

Resolving lexical ambiguities is key component of skilled language comprehension. Without the ability to access the contextually appropriate, intended meaning for each word we encounter, accurate communication between individuals would be impossible. Evidence from behavioural experiments has indicated that retrieval of word meanings can be modelled within a distributed connectionist framework in which words compete to produce coherent patterns of activation across an array of semantic 'units'. Ambiguity between multiple meanings can interfere with this process, making it more challenging to retrieve the meanings of these words compared to unambiguous words. However, once a sentence context is provided that strongly supports just one of a word's possible meanings, then readers and listeners are able 
to make use of executive function control processes to select the most likely meanings, and if necessary reinterpret the sentence in the light of subsequent information.

\section{Acknowledgements}

Thanks to Becky Gilbert for providing the terrible pun about the fish. And more importantly for her insightful comments on a draft version of this chapter.

Reference List

Key Stage 2 English Reading Booklet (2016). Key Stage 2 English Reading Booklet [Electronic version]. Available: https://www.gov.uk/government/publications/key-stage-2-tests-2016-englishreading-test-materials

Armstrong, B. C. \& Plaut, D. C. (2016). Disparate semantic ambiguity effects from semantic processing dynamics rather than qualitative task differences. Language, Cognition and Neuroscience, 31, 940-966.

Armstrong, B. C., Tokowicz, N., \& Plaut, D. C. (2012). eDom: Norming software and relative meaning frequencies for 544 English homonyms. 44, 10151027. 
Azuma, T. \& Van Orden, G. C. (1997). Why SAFE is better than FAST: The relatedness of a word's meanings affects lexical decision times. Journal of Memory and Language, 36, 484-504.

Bates, E., Devescovi, A., \& Wulfeck, B. (2001). Psycholinguistics: A crosslanguage perspective. Annual Review of Psychology, 52, 369-396.

Bedny, M., McGill, M., \& Thompson-Schill, S. L. (2008). Semantic adaptation and competition during word comprehension. 18, 2574-2585.

Bekinschtein, T. A., Davis, M. H., Rodd, J. M., \& Owen, A. M. (2011). Why clowns taste funny: the relationship between humor and semantic ambiguity. Journal of Neuroscience, 31, 9665-9671.

Beretta, A., Fiorentino, R., \& Poeppel, D. (2005). The effects of homonymy and polysemy on lexical access: An MEG study. Cognitive Brain Research, 24, 5765.

Blank, A. (1999). Why do new meanings occur? A cognitive typology of the motivations for lexical semantic change. In A.Blank \& P. Koch (Eds.), Historical Semantics and Cognition (pp. 61-90). Berlin/New York: Mouton de Gruyter.

Borowsky, R. \& Masson, M. E. J. (1996). Semantic ambiguity effects in word identification. Journal of Experimental Psychology: Learning Memory and Cognition, 22, 63-85.

Casenhiser, D. M. (2005). Children's resistance to homonymy: An experimental study of pseudohomonyms. Journal of Child Language, 32, 319-343. 
Clark, H. H. \& Gerrig, R. J. (1983). Understanding old words with new meanings. Journal of Verbal Learning and Verbal Behavior, 22, 591-608.

Cruse, A. D. (1986). Lexical Semantics. Cambridge, UK: Cambridge University Press.

Dautriche, I. \& Chemla, E. (2016). What homophones say about words. PLoS ONE, 11.

Dautriche, I., Chemla, E., \& Christophe, A. (2016). Word Learning: Homophony and the Distribution of Learning Exemplars. Language Learning and Development, 12, 231-251.

Davis, M. H., Coleman, M. R., Absalom, A. R., Rodd, J. M., Johnsrude, I. S., Matta, B. F. et al. (2007). Dissociating speech perception and comprehension at reduced levels of awareness. Proc.NatI.Acad.Sci.U.S.A, 104, 16032-16037.

Dijkstra, T., Grainger, J., \& Van Heuven, W. J. B. (1999). Recognition of Cognates and Interlingual Homographs: The Neglected Role of Phonology. 41, 496518.

Doherty, M. J. (2004). Children's difficulty in learning homonyms. Journal of Child Language, 31, 203-214.

Duffy, S. A., Morris, R. K., \& Rayner, K. (1988). Lexical ambiguity and fixation times in reading. Journal of Memory and Language, 27, 429-446.

Fang, X., Perfetti, C., \& Stafura, J. Learning new meanings for known words: biphasic effects of prior knowledge. Language, Cognition and Neuroscience, (in press). 
Frisson, S. \& Pickering, M. (2007). The processing of familiar and novel senses of a word: Why reading Dickens is easy but reading Needham can be hard. Language and Cognitive Processes, 22, 595-613.

Gernsbacher, M. A. \& Faust, M. E. (1991). The mechanism of suppression: a component of general comprehension skill. Journal of Experimental Psychology: Learning Memory and Cognition, 17, 245-262.

Gernsbacher, M. A., Varner, K. R., \& Faust, M. E. (1990). Investigating differences in general comprehension skill. Journal of Experimental Psychology: Learning Memory and Cognition, 16, 430-445.

Hagoort, P. (2005). Unification in language and the brain. In A.Cutler (Ed.), Twenty-First Century Psycholinguistics: Four Cornerstones (pp. 157-172). Lawrence Erlbaum Associates.

Hagoort, P. \& Indefrey, P. (2014). The neurobiology of language beyond single words. Annual Review of Neuroscience, 37, 347-362.

Henderson, L., Snowling, M., \& Clarke, P. (2013). Accessing, Integrating, and Inhibiting Word Meaning in Poor Comprehenders. Scientific Studies of Reading, 17, 177-198.

Hino, Y., Pexman, P. M., \& Lupker, S. J. (2006). Ambiguity and relatedness effects in semantic tasks: Are they due to semantic coding? Journal of Memory and Language, 55, 247-273. 
Hino, Y. \& Lupker, S. J. (1996). Effects of polysemy in lexical decision and naming - an alternative to lexical access accounts. Journal of Experimental Psychology: Human Perception and Performance, 22, 1331-1356.

Jastrzembski, J. E. (1981). Multiple meanings, number of related meanings, frequency of occurrence, and the lexicon. Cognitive Psychology, 13, 278-305.

Joordens, S. \& Besner, D. (1994). When banking on meaning is not (yet) money in the bank - explorations in connectionist modeling. Journal of Experimental Psychology: Learning Memory and Cognition, 20, 1051-1062.

Kambe, G., Rayner, K., \& Duffy, S. A. (2001). Global context effects on processing lexically ambiguous words: evidence from eye fixations. Memory and Cognition, 29, 363-72.

Kan, I. P. \& Thompson-Schill, S. L. (2004). Effect of name agreement on prefrontal activity during overt and covert picture naming. Cognitive, Affective and Behavioral Neuroscience, 4, 43-57.

Kawamoto, A. H., Farrar, W. T., \& Kello, C. T. (1994). When two meanings are better than one: Modeling the ambiguity advantage using a recurrent distributed network. Journal of Experimental Psychology: Human Perception and Performance, 20, 1233-1247.

Kellas, G., Ferraro, F. R., \& Simpson, G. B. (1988). Lexical ambiguity and the timecourse of attentional allocation in word recognition. Journal of Experimental Psychology: Human Perception and Performance, 14, 601-609. 
Khanna, M. M. \& Boland, J. E. (2010). Children's use of language context in lexical ambiguity resolution. Quarterly Journal of Experimental Psychology, 63, 160193.

Klein, D. E. \& Murphy, G. L. (2001). The Representation of Polysemous Words. Journal of Memory and Language, 45, 259-282.

Klepousniotou, E. \& Baum, S. R. (2007). Disambiguating the ambiguity advantage effect in word recognition: An advantage for polysemous but not homonymous words. Journal of Neurolinguistics, 20, 1-24.

MacDonald, M. C., Pearlmutter, N. J., \& Seidenberg, M. S. (1994). The lexical nature of syntactic ambiguity resolution. Psychological Review, 101, 676-703.

Marian, V., Bartolotti, J., Chabal, S., \& Shook, A. (2012). Clearpond: Crosslinguistic easy-access resource for phonological and orthographic neighborhood densities. PLOS ONE, 7.

Mason, R. A. \& Just, M. A. (2007). Lexical ambiguity in sentence comprehension. Brain Research, 1146, 115-127.

Mazzocco, M. M. M. (1997). Children's interpretations of homonyms: A developmental study. Journal of Child Language, 24, 441-467.

McClelland, J. L. \& Elman, J. L. (1986). The TRACE model of speech perception. Cognitive Psychology, 18, 1-86.

McClelland, J. L. \& Rumelhart, D. E. (1981). An interactive activation model of context effects in letter perception: Part 1. An account of basic findings. Psychological Review, 88, 375-407. 
McRae, K., Spivey-Knowlton, M. J., \& Tanenhaus, M. K. (1998). Modeling the influence of thematic fit (and other constraints) in on-line sentence comprehension. Journal of Memory and Language, 38, 283-312.

Millis, M. L. \& Button, S. B. (1989). The effect of polysemy on lexical decision time: now you see it, now you don't. Memory and Cognition, 17, 141-147.

Miyake, A., Just, M. A., \& Carpenter, P. A. (1994). Working Memory Constraints on the Resolution of Lexical Ambiguity: Maintaining Multiple Interpretations in Neutral Contexts. Journal of Memory and Language, 33, 175-202.

Norbury, C. F. (2005). Barking up the wrong tree? Lexical ambiguity resolution in children with language impairments and autistic spectrum disorders. Journal of Experimental Child Psychology, 90, 142-171.

Novick, J. M., Kan, I. P., Trueswell, J. C., \& Thompson-Schill, S. L. (2009). A case for conflict across multiple domains: Memory and language impairments following damage to ventrolateral prefrontal cortex. Cognitive Neuropsychology, 26, $527-567$.

Novick, J. M., Trueswell, J. C., \& Thompson-Schill, S. L. (2005). Cognitive control and parsing: Reexamining the role of Broca's area in sentence comprehension. Cognitive Affective \& Behavioral Neuroscience, 5, 263-281.

Onifer, W. \& Swinney, D. A. (1981). Accessing lexical ambiguities during sentence comprehension - effects of frequency of meaning and contextual bias. Memory and Cognition, 9, 1-39. 
Parks, R., Ray, J., \& Bland, S. (1998). Wordsmyth English DictionaryThesaurus. University of Chicago, http://www.wordsmyth.net/ [Electronic version].

Pexman, P. M. \& Lupker, S. J. (1999). Ambiguity and visual word recognition: can feedback explain both homophone and polysemy Effects? Canadian Journal of Experimental Psychology, 53, 323-334.

Piantadosi, S. T., Tily, H., \& Gibson, E. (2012). The communicative function of ambiguity in language. 122, 280-291.

Poort, E. D., Warren, J. E., \& Rodd, J. M. (2016). Recent experience with cognates and interlingual homographs in one language affects subsequent processing in another language. Bilingualism, 19, 206-212.

Rabagliati, H., Marcus, G. F., \& Pylkkänen (2010). Shifting senses in lexical semantic development. Cognition, 117, 17-37.

Rabagliati, H., Pylkkänen, L., \& Marcus, G. F. (2013). Top-down influence in young children's linguistic ambiguity resolution. Developmental Psychology, 49, 10761089.

Rodd, J. M., Berriman, R., Landau, M., Lee, T., Ho, C., Gaskell, M. G. et al. (2012). Learning new meanings for old words: effects of semantic relatedness. Memory and Cognition, 40, 1095-1108.

Rodd, J. M., Cai, Z. G., Betts, H. N., Hanby, B., Hutchinson, C., \& Adler, A. (2016). The impact of recent and long-term experience on access to word meanings: Evidence from large-scale internet-based experiments. Journal of Memory and Language, 87, 16-37. 
Rodd, J. M., Davis, M. H., \& Johnsrude, I. S. (2005). The neural mechanisms of speech comprehension: fMRI studies of semantic ambiguity. Cerebral Cortex, 15, 1261-1269.

Rodd, J. M., Gaskell, M. G., \& Marslen-Wilson, W. D. (2004). Modelling the effects of semantic ambiguity in word recognition. Cognitive Science, 28, 89-104.

Rodd, J. M., Johnsrude, I. S., \& Davis, M. H. (2010). The role of domaingeneral frontal systems in language comprehension: Evidence from dual-task interference and semantic ambiguity. Brain and Language, 115, 182-188.

Rodd, J. M., Johnsrude, I. S., \& Davis, M. H. (2012). Dissociating frontotemporal contributions to semantic ambiguity resolution in spoken sentences. Cerebral Cortex, 22, 1761-1773.

Rodd, J. M., Longe, O. A., Randall, B., \& Tyler, L. K. (2010). The functional organisation of the fronto-temporal language system: Evidence from syntactic and semantic ambiguity. Neuropsychologia, 48, 1324-1335.

Rodd, J. M., Lopez Cutrin, B., Kirsch, H., Millar, A., \& Davis, M. H. (2013). Long-term priming of the meanings of ambiguous words. Journal of Memory and Language, 68, 180-198.

Rodd, J. M., Vitello, S., Woollams, A. M., \& Adank, P. (2015). Localising semantic and syntactic processing in spoken and written language comprehension: An Activation Likelihood Estimation meta-analysis. Brain and Language, 141, 89-102.

Rodd, J. M. (2004). The effect of semantic ambiguity on reading aloud: A twist in the tale. Psychonomic Bulletin and Review, 11, 440-445. 
Rodd, J. M., Gaskell, M. G., \& Marslen-Wilson, W. D. (2002). Making sense of semantic ambiguity: Semantic competition in lexical access. Journal of Memory and Language, 46, 245-266.

Rorden, C. \& Brett, M. (2000). Stereotaxic display of brain lesions. Behavioural Neurology, 12, 191-200.

Rubenstein, H., Garfield, L., \& Millikan, J. A. (1970). Homographic Entries in the Internal Lexicon. Journal of Verbal Learning and Verbal Behavior, 9, 487-494.

Schepens, J., Dijkstra, T., \& Grootjen, F. (2012). Distributions of cognates in Europe as based on Levenshtein distance. Bilingualism, 15, 157-166.

Seidenberg, M. S., Tanenhaus, M. K., Leiman, J. M., \& Bienkowski, M. (1982). Automatic access of the meanings of ambiguous words in context - Some limitations of knowledge-based processing. Cognitive Psychology, 14, 489-537.

Simpson, G. B. \& Kang, H. (1994). Inhibitory processes in the recognition of homograph meanings. In Inhibitory Processes in Attention, Memory, and Language (pp. 359-381). New York: Academic Press.

Srinivasan, M. \& Snedeker, J. (2011). Judging a book by its cover and its contents: The representation of polysemous and homophonous meanings in fouryear-old children. Cognitive Psychology, 62, 245-272.

Storkel, H. L. \& Maekawa, J. (2005). A comparison of homonym and novel word learning: The role of phonotactic probability and word frequency. Journal of Child Language, 32, 827-853. 
Storkel, H. L., Maekawa, J., \& Aschenbrenner, A. J. (2013). The effect of homonymy on learning correctly articulated versus misarticulated words. Journal of Speech, Language, and Hearing Research, 56, 694-707.

Swinney, D., Onifer, W., Prather, P., \& Hirshkowitz, M. (1979). Semantic facilitation across modalities in the processing of individual words and sentences. Memory and Cognition, 7, 159-165.

Swinney, D. A. (1979). Lexical access during sentence comprehension: (re)consideration of context effects. Journal of Verbal Learning and Verbal Behavior, $18,645-659$.

Tabossi, P. (1988). Accessing lexical ambiguity in different types of sentential contexts. Journal of Memory and Language, 27, 324-340.

Tabossi, P. \& Zardon, F. (1993). Processing ambiguous words in context. Journal of Memory and Language, 32, 359-372.

Tahmasebi, A. M., Davis, M. H., Wild, C. J., Rodd, J. M., Hakyemez, H., Abolmaesumi, P. et al. (2012). Is the link between anatomical structure and function equally strong at all cognitive levels of processing? Cerebral Cortex, 22, 1593-1603.

Thompson-Schill, S. L., D'Esposito, M., Aguirre, G. K., \& Farah, M. J. (1997). Role of left inferior prefrontal cortex in retrieval of semantic knowledge: A reevaluation. Proceedings of the National Academy of Sciences of the United States of America, $94,14792-14797$.

Twilley, L. C. \& Dixon, P. (2000). Meaning resolution processes for words: a parallel independent model. Psychonomic Bulletin and Review, 7, 49-82. 
Twilley, L. C., Dixon, P., Taylor, D., \& Clark, K. (1994). University of Alberta norms of relative meaning frequency for 566 homographs. Memory and Cognition, 22, 111-126.

van der Schoot, M., Vasbinder, A. L., Horsley, T. M., Reijntjes, A., \& van Lieshout, E. C. D. M. (2009). Lexical Ambiguity Resolution in Good and Poor Comprehenders: An Eye Fixation and Self-Paced Reading Study In Primary School Children. Journal of Educational Psychology, 101, 21-36.

Vitello, S. \& Rodd, J. M. (2015). Resolving Semantic Ambiguities in Sentences: Cognitive Processes and Brain Mechanisms. Linguistics and Language Compass, 9, 391-405.

Vitello, S., Warren, J. E., Devlin, J. T., \& Rodd, J. M. (2014). Roles of frontal and temporal regions in reinterpreting semantically ambiguous sentences. Frontiers in Human Neuroscience, 8.

Vitevitch, M. S. (2012). What do foreign neighbors say about the mental lexicon? Bilingualism, 15, 167-172.

Whitney, C., Kirk, M., O'Sullivan, J., Lambon Ralph, M. A., \& Jefferies, E. (2011). The neural organization of semantic control: TMS evidence for a distributed network in left inferior frontal and posterior middle temporal gyrus. Cerebral Cortex, $21,1066-1075$.

Zempleni, M. Z., Renken, R., Hoeks, J. C., Hoogduin, J. M., \& Stowe, L. A. (2007). Semantic ambiguity processing in sentence context: Evidence from eventrelated fMRI. Neuroimage., 34, 1270-1279. 
